\section{SPORT ZUR PRÄVENTION}

\section{„Bewegungshäppchen“ für zwischendurch}

- Bevölkerungsstudien in den USA und in Europa lassen große Defizite im Bereich der körperlichen Aktivität erkennen. In den USA haben die Fachgesellschaften deshalb neue Leitlinien formuliert, die nicht mehr nur ein zwei- oder besser ein dreimaliges Trainingsprogramm pro Woche von eineinhalb Stunden propagieren, sondern zusätzlich tägliche kleinere Trainingseinheiten, soge- nannte „Bewegungshäppchen“ vorsehen.

Neben der Inaktivität ist nach Prof. Predel, Köln, vor allem die abdominelle Adipositas ein gewichtiger kardiovaskulärer Risikofaktor. XXL-Hypertoniker sind als Hochrisikopatienten anzusehen und brauchen ein striktes Risiko-Management, wobei analog zu Diabetikern Blutdruckwerte unter 130/80 $\mathrm{mmHg}$ anzustreben sind.

\title{
MASSENWARE ANABOLIKA
}

\section{Doping ein Volkssport?}

— Prof. Dr. M. Thevis, Leiter des Zentrums für Präventive Dopingforschung an der Sporthochschule Köln, geht davon aus, dass in Deutschland auch im Breitensport „flächendeckend“ gedopt wird. Anabole Wirksubstanzen kämen eimerweise ins Land. Und bei Weitem nicht alles, was geschluckt, gespritzt oder infundiert wird, ist auch nachzuweisen, wenngleich die Analysemethoden ständig verbessert werden. Aber etwa modifizierte Steroide mit unbekanntem Molekulargewicht werden nicht erfasst, meint Thevis. Im Breitensport ohnehin nicht, denn da werden in der Regel keine Kontrollen durchgeführt.

Diskutiert wird, ob eine Meldepflicht eingeführt werden soll. Bislang ist der Arzt an seine Schweigepflicht gebunden.

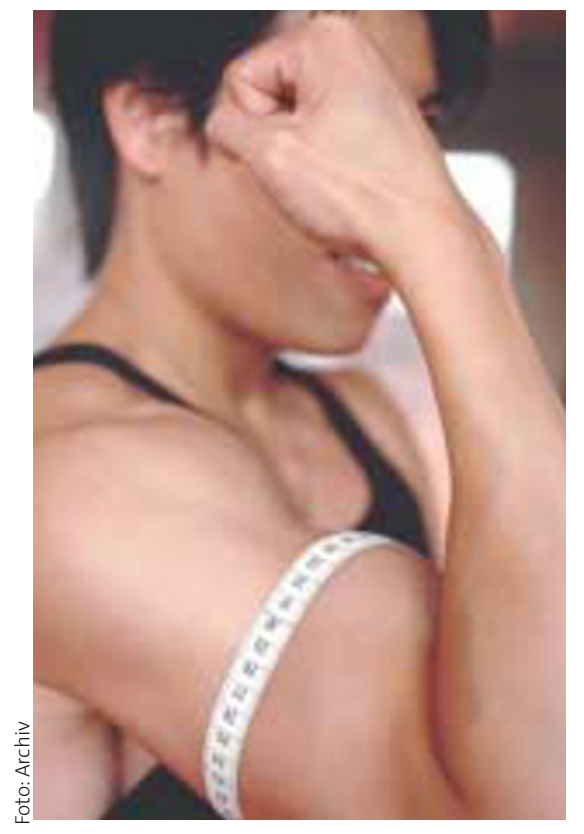

Alles antrainiert? Bei solchen Muskeln sind Zweifel erlaubt.

\section{KRANKENHÄUSER KEIN VORBILD}

\section{Bettruhe statt Bewegung}

— Der Präsident der Gesellschaft der deutschen Sportärzte, Prof. H. Löllgen, kritisierte, dass die sportmedizinischen Erkenntnisse nur unzureichend im medizinischen Alltag umgesetzt würden. Körperliche Aktivität sollte wesentlicher Bestandteil der Therapie und Rehabilitation bei Herzkrankheiten, Lungenkrankheiten, Krebsleiden, psychischen Erkrankungen sowie bei Osteoporose sein. Dabei müsste die regelmäßige körperliche Aktivität bereits im Krankenhaus einsetzen. Aber: „Hier werden die Patienten viel zu oft ins Bett gelegt oder geschont, wo Aktivität und Bewegung angezeigt wären.“

\section{NACH DEN DOPINGSKANDALEN}

\section{Bild der Sportmedizin beschädigt}

- Die Sportärzte wehren sich heftig dagegen, pauschal als Dopingärzte diffamiert zu werden. Dass Doping bei Leistungssportlern, aber auch im Breitensport kategorisch abzulehnen ist, darüber ließ der Verbandspräsident Prof. Herbert Löllgen, Remscheid, nicht den geringsten Zweifel aufkommen. Es sei zwar bedauerlich, dass sich deutsche Sportärzte schuldig gemacht haben, aber die Konsequenzen wurden bzw. werden gezogen. Sportärzte sollten für ihre Leistungen angemessen bezahlt werden, sie dürfen sich aber weder vom Verband noch von den Sportlern vereinnahmen lassen, betonte Olympiaarzt Prof. Dr. Wilfried Kindermann. Das große „Blutgeschäft“ bei der Tour de France sei im Übrigen nicht von einem Sportarzt, sondern von dem spanischen Gynäkologen Dr. Fuentes zu verantworten.

Aufgabe der Sportmedizin sei nicht nur die Betreuung der Leistungssportler, sondern auch die Prävention in der ganzen Bevölkerung, denn: „Körperliche Aktivität ist unverzichtbar für die Gesundheit.“

DR. MED. JOCHEN AUMILLER .

Quelle: 40. Deutscher Sportärztekongress, Köln, 27.-29. September 2007 